\title{
Evaluaciones microcalorimétricas no-isotermas en aleaciones de Cu-9Ni-5,5Sn templadas y deformadas en frío( ${ }^{(*)}$
}

\author{
E. Donoso*, M.J. Diánez** y J.M. Criado**
}

\begin{abstract}
Resumen
Se ha estudiado por calorimetría diferencial de barrido (DSC) y medidas de microdureza, el comportamiento durante el recocido de una aleación Cu-9 \% Ni-5,5 \% Sn templada y deformada por laminación en frio al 50 \%, respectivamente. Se ha concluido que durante el tratamiento térmico de la muestra templada tiene lugar un aumento de la microdureza atribuido a la precipitación de una fase $\gamma^{\prime}$ de composición $\left(\mathrm{Cu}_{\mathrm{x}} \mathrm{Ni}_{1-\mathrm{x}}\right)_{3} \mathrm{Sn}$. Sin embargo, no se ha observado un aumento de dureza durante el recocido de la muestra deformada en frío. Este comportamiento se ha interpretado considerando que las dislocaciones generadas por la laminación en frío, favorecen la segregación de soluto (níquel y estaño) hacia las dislocaciones en lugar de la formación de la fase $\left(\mathrm{Cu}_{\mathrm{x}} \mathrm{Ni}_{1-\mathrm{x}}\right)_{3} \mathrm{Sn}$. Tanto el análisis teórico de la entalpía determinada para esta etapa como los valores de los coeficientes $n$ de Avrami-Erofeev determinados a partir de un análisis cinético apoyan esta interpretación.
\end{abstract}

\section{Non-isothermal microcalorimetric evaluations in quenched and in cold-rolled Cu-9Ni-5.5Sn alloys}

\begin{abstract}
The thermal aging of both a quenched and a cold rolled homogeneous supersaturated $\mathrm{Cu}-9 \%$ wt $\mathrm{Ni}-5.5 \mathrm{wt} \% \mathrm{Sn}$ alloy has been studied from differential scanning calorimetry (DSC) and microhardness measurements. An increase of the hardness during the aging of the quenched sample, because of the precipitation of a $\gamma^{\prime}$ phase, takes place. On the contrary, no hardness increase was observed during the aging of the cold rolled sample. A theoretical analysis of the enthalpy determined from the first DSC exothermic peak suggests that a segregation of the solute towards the dislocations occurs during the aging of the cold rolled alloy. The values of the $n$ Avrami-Erofeev coefficients estimated from the kinetic analysis supports the above interpretations.
\end{abstract}

Keywords

Copper; $\mathrm{Cu}-\mathrm{Ni}$-Si; Precipitation; Microcalorimetry.

\section{INTRODUCCION}

El desarrollo de aleaciones ternarias de alta resistencia mecánica ricas en cobre, ha sido objeto de numerosos estudios en la última década ${ }^{[1-3]}$. En la mayor parte de ellos se ha considerado que el incremento de la resistencia mecánica se debe a la formación de precipitados binarios y/o ternarios de tamaño nanométrico que contribuyen al anclaje de las dislocaciones, con el consiguiente aumento de la dureza. En condiciones de equilibrio, las aleaciones de $\mathrm{Cu}-\mathrm{Ni}-\mathrm{Sn}$ ricas en cobre son soluciones sólidas con estructura tipo fase $\alpha$, siendo posible obtener soluciones sóli- das sobresaturadas a temperatura ambiente por templado a temperaturas superiores a la de equilibrio. Con tratamientos de envejecimiento adecuados, estas aleaciones desarrollan una excelente combinación de resistencia mecánica, alta conductividad eléctrica y alta resistencia a la corrosión. Los estudios realizados sobre el mecanismos de endurecimiento de las aleaciones de $\mathrm{Cu}-\mathrm{Ni}$ - $\mathrm{Sn}^{[4-13]}$, señalan que el aumento de la resistencia mecánica tiene lugar a través de una descomposición espinodal seguida de la precipitación de partículas metaestables $\gamma^{\prime}$, con estructura $\mathrm{DO}_{22}$ y composición estequiométrica $\left(\mathrm{Cu}_{\mathrm{x}} \mathrm{Ni}_{1-\mathrm{x}}\right)_{3} \mathrm{Sn}$, que finalmente se convierte en la fase de equilibrio $\gamma$,

\footnotetext{
(•) Trabajo recibido el día 03 de agosto de 2011 y aceptado en su forma final el día 08 de noviembre de 2011.

* Universidad de Chile, Facultad de Ciencias Físicas y Matemáticas, Departamento de Ciencia de los Materiales, Av. Tupper 2069, Piso 2, casilla 2777, Santiago-Chile, e-mail: edonoso@ing.uchile.cl

** Instituto de Ciencias de Materiales de Sevilla, Américo Vespucio 49, Isla de la Cartuja, 41092 Sevilla-España, e-mail: jmcriado@icmse.csic.es
} 
de la misma estequiometría y estructura cristalina tipo $\mathrm{DO}_{3}$. Algunos autores encuentran que las partículas con estructura $\mathrm{DO}_{22}$ son las responsables del endurecimiento por precipitación en la matriz de cobre $^{[5,10 \text { y } 13]}$. Por otra parte, es sabido que en soluciones sólidas de cobre deformadas en frío y recocidas a temperaturas inferiores a la de recristalización, tiene lugar un bloqueo de las dislocaciones en el que la migración del soluto hacia estas juega un papel importante ${ }^{[14]}$. En el caso de que este fenómeno origine una ramificación de las dislocaciones podría dar lugar a un notable aumento de la dureza, denominado endurecimiento por recocido ${ }^{[15-21]}$. En el presente trabajo se pretende obtener información adicional sobre el mecanismo de endurecimiento a partir del análisis cinético del proceso de cristalización y del análisis teórico de los valores determinados para la entalpía de cristalización de una aleación de composición Cu-9 \% Ni-5,5. \% Sn, tanto deformada como templada.

\section{PROCEDIMIENTO EXPERIMENTAL}

La aleación utilizada en este estudio se preparó por fusión en un horno de inducción en atmósfera inerte $(\mathrm{Ar})$, a partir de una mezcla en proporciones estequiométricas de cobre electrolítico (99,95\% de pureza), níquel y estaño de alta pureza. El lingote obtenido se recoció a $1.073 \mathrm{~K}$ durante 24 h (en atmósfera inerte) a fin de homogeneizarlo. Posteriormente, el material se sometió a tres sucesivas laminaciones en frío, con recocidos intermedios de $1 \mathrm{~h}$ a $1.073 \mathrm{~K}$, hasta su reducción a planchas de $4 \mathrm{~mm}$ de espesor. Después de la última laminación se sometió la muestra a un último recocido de $1 \mathrm{~h}$ a $1.073 \mathrm{~K}$ seguido de un temple (aleación templada). Se preparó una segunda muestra sometiendo a la primera a una deformación por laminación en frío del 50 \% (aleación deformada). El análisis químico realizado a las muestra indica que su composición es $\mathrm{Cu}-9$ \% Ni-5,5 \% Sn en peso.

Se ha utilizado un equipo de calorimetría diferencial de barrido (DSC) TA Instruments Q10. Los diagramas de DSC se han registrado a diversas velocidades de calentamiento bajo un flujo de argón de $10^{-4} \mathrm{~m}^{3} \mathrm{~min}^{-1}$. A fin de aumentar la precisión de las medidas, se utilizó como referencia un disco de cobre de alta pureza recocido durante un largo período. La corrección de la línea base se llevó a cabo por el método previamente descrito en publicaciones anteriores $^{[22}$ y 23$]$.

Los diagramas de difracción de rayos $\mathrm{X}$ se han registrado en un difractómetro PANanalytical X'pert Pro, utilizando radiación $\mathrm{Cu} \mathrm{K}_{\alpha}$. Los parámetros reticulares se han calculado por ajuste de mínimos cuadrados mediante el programa Cell-Ref.
Las medidas de microdureza Vickers se efectuaron a temperatura ambiente en un microdurómetro Duramin -1/-2 Struers de alta precisión, aplicando una carga de 1,96 $\mathrm{N}$ durante $10 \mathrm{~s}$.

\section{RESULTADOS Y DISCUSIÓN}

\subsection{Curvas calorimétricas}

En la figura 1 se incluyen los diagramas de difracción de rayos $\mathrm{X}$ de las muestras templadas y deformadas, respectivamente, que ponen claramente de manifiesto que en ambos casos se observa una única fase de estructura tipo $\mathrm{Cu}-\alpha$. El parámetro reticular determinado para la muestra templada es $a=3,641(0,002) \AA$. Este valor coincide con el determinado en una publicación anterior ${ }^{[12]}$ para una aleación homogénea $\mathrm{Cu}-\mathrm{Ni}$-Sn de análoga composición. El valor del parámetro reticular determinado para la aleación deformada al $50 \%$ ha sido $a=3,637(0,002) \AA$. La ligera disminución sufrida por el parámetro reticular tras la laminación en frío sugiere que ha tenido lugar una segregación parcial del estaño hacia las dislocaciones creadas por la laminación al 50 \%. Por otra parte, el ensanchamiento de los picos de difracción observados en la muestra laminada pone de manifiesto que se ha producido una disminución del grado de cristalinidad de la muestra como consecuencia de los defectos creados durante el tratamiento mecánico. Este comportamiento se observa claramente en la ventana insertada en la figura 1 en la que se comparan las anchuras del pico de difracción de los planos (311) de las muestras templadas y deformadas en frío. Puede observarse que las reflexiones $\mathrm{K}_{\alpha 1}$ y $\mathrm{K}_{\alpha 2}$ del pico (311) se discriminan claramente en la muestra templada, mientras que en la muestra deformada han quedado completamente enmascaradas por el ensanchamiento de los picos de difracción.

Los diagramas de DSC registrados a velocidades de calentamiento comprendidas entre $0,083 \mathrm{Ks}^{-1} \mathrm{y}$ $0,667 \mathrm{Ks}^{-1}$ para las muestras de aleación templada y aleación deformada se incluyen en las figuras 2 y 3 , respectivamente. Puede observarse que en ambos materiales se observan dos picos exotérmicos que se designarán de aquí en adelante como etapas 1 y 2 para la aleación templada y etapas 3 y 4 para la aleación deformada. El hecho de que las temperaturas de los picos de DSC se desplacen a temperaturas más altas al aumentar la velocidad de calentamiento pone de manifiesto el control cinético del proceso ${ }^{[24]}$. Las entalpías correspondientes a dichas etapas se han determinado a partir de las áreas de los picos asociadas a las mismas en las curvas de DSC. En el caso de la muestra templada, la deconvolución de los 


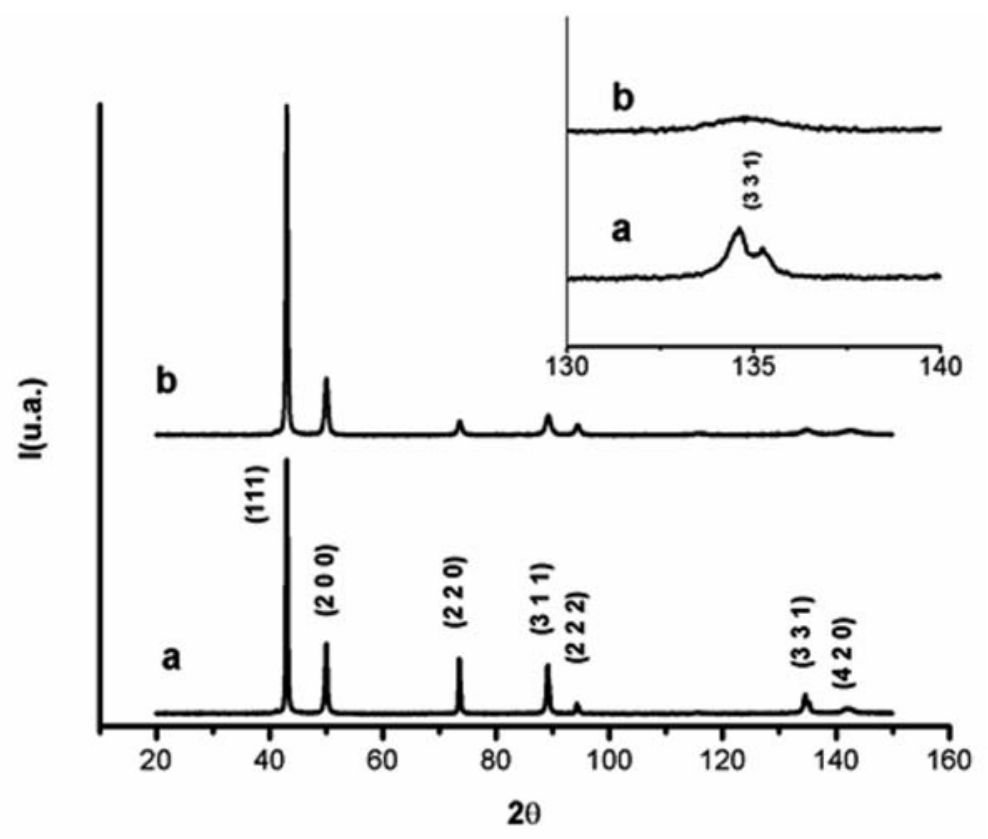

Figura 1. Diagramas de difracción de rayos $X$ de la aleación templada (a) y de la aleación deformada (b).

Figure 1. X ray diffraction patterns of quenched alloy (a) and of cold-rolled alloy (b).

picos se llevó a cabo mediante el ajuste por un algoritmo Frazer-Suzuki como se indica en una publicación anterior ${ }^{[25]}$. En la figura 4 se muestra, a título de ejemplo, el ajuste de la curva de DSC obtenida a una velocidad de calentamiento de $0,167 \mathrm{Ks}^{-1}$. Los valores promedios obtenidos para la entalpía asociada a cada etapa a partir de la serie de diagramas de DSC incluidos en las figuras 2 y 3 , respectivamente, se incluyen en la tabla I. La mayor energía asociada a los picos exotérmicos de la aleación deformada con respecto a la aleación templada podría adscribirse a la energía extra almacenada por las dislocaciones generadas durante la deformación en frío. Con objeto de analizar la posible relación existente entre los procesos exotérmicos observados y las propiedades mecánicas de las dos aleaciones estudiadas, se han realizado medidas de la microdureza de Vickers de muestras obtenidas deteniendo a temperaturas seleccionadas los diagramas de DSC registrados para ambas muestras a una velocidad de $0,167 \mathrm{Ks}^{-1}$. Las temperaturas a las que se han extraído las muestras del aparato de DSC así como la microdureza Vickers determinadas para las mismas se muestran en la tabla II. Estos resultados ponen de manifiesto que la deformación por laminación en frío al 50 \% de la aleación $\mathrm{Cu}-9$ \% Ni-5,5 \% Sn produce, como era de esperar, un considerable incremento de la dureza debido a las dislocaciones generadas por laminación.

Tabla I. Medidas energéticas y cinéticas asociadas a las diferentes etapas observadas en Cu-9 \% Ni-5,5\% Sn

Table I. Energetic and kinetics measurements associated with the different stages observed in Cu-9\% wt Ni-5.5 wt Sn

\begin{tabular}{lcccc}
\hline \multicolumn{1}{c}{ Etapas } & $\mathbf{1}$ & $\mathbf{2}$ & $\mathbf{3}$ & $\mathbf{4}$ \\
\hline$\Delta \mathrm{H}\left(\mathrm{J} \mathrm{mol}^{-1}\right)$ & $330 \pm 9$ & $510 \pm 12$ & $440 \pm 10$ & $635 \pm 14$ \\
$\mathrm{E}\left(\mathrm{kJ} \mathrm{mol}^{-1}\right)$ & 103 & 123 & 63 & - \\
$\mathrm{A}\left(\mathrm{s}^{-1}\right)$ & $4 \times 108$ & $1 \times 109$ & $6 \times 106$ & - \\
$\mathrm{n}$ & 1,1 & 1,6 & 0,6 & - \\
\hline
\end{tabular}




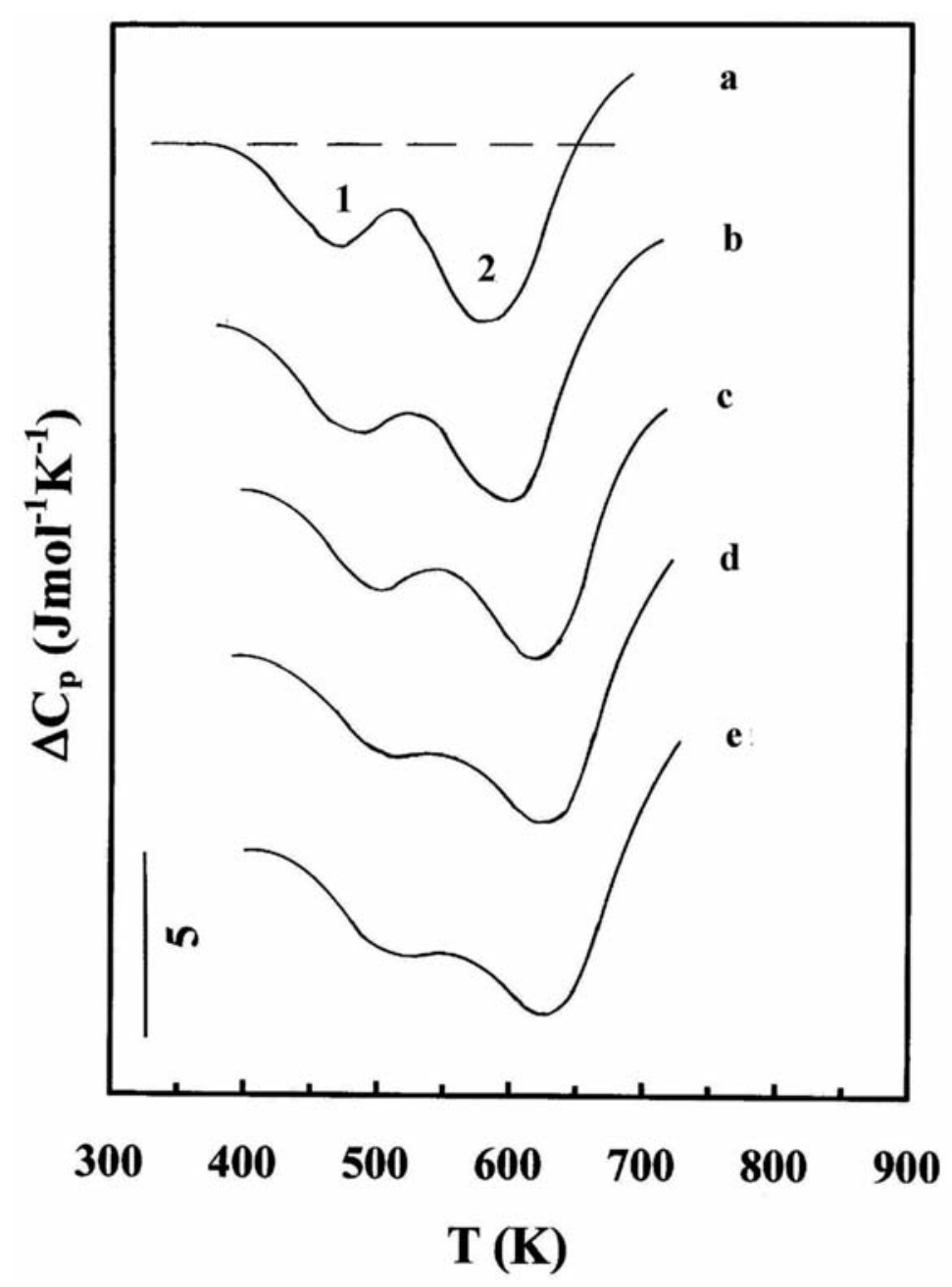

Figura 2. Curvas DSC para Cu- $9 \% \mathrm{Ni}-5,5 \%$ templado, a las velocidades de calentamiento lineal de: a) $0,083 \mathrm{Ks}^{-1}$, b) $0,167 \mathrm{~K} \mathrm{~s}^{-1}$, c) $0,333 \mathrm{~K} \mathrm{~s}^{-1}$, d) $0,50 \mathrm{~K} \mathrm{~s}^{-1}$, y e) $0,667 \mathrm{~K} \mathrm{~s}^{-1}$.

Figure 2. DSC curves for quenched Cu-9 wt. \% Ni-5.5 wt. \%, at the heating rates of: a) $0.083 \mathrm{Ks}^{-1}$, b) $0.167 \mathrm{~K} \mathrm{~s}^{-1}$, c) $0.333 \mathrm{~K} \mathrm{~s}^{-1}$, d) $0.50 \mathrm{~K} \mathrm{~s}^{-1}$, and e) $0.667 \mathrm{Ks}^{-1}$.

Estos resultados ponen también de manifiesto que en el caso de la aleación templada tiene lugar un notable aumento de la dureza asociado a la aparición de los picos exotérmicos, mientras que en el caso de la aleación deformada el segundo pico exotérmico está asociado, por el contrario, a una disminución de la dureza. El aumento de la dureza experimentado durante el calentamiento de la muestra templada podría atribuirse a la formación de precipitados dispersos en la matriz de cobre. Los estudios estructurales previamente realizados en la bibliografía ${ }^{[4,7,8,11-13]}$ sobre aleaciones de composición análoga a la estudiada en este trabajo apoyan esta interpretación, dado que se ha reportado la formación de fases del tipo $\left(\mathrm{Cu}_{\mathrm{x}} \mathrm{Ni}_{1-\mathrm{x}}\right)_{3} \mathrm{Sn}$ en muestras recocidas en el rango de temperaturas en que se observan los picos exotérmicos en la figura 2. Por
Tabla II. Valores de microdureza Vickers medidas a temperatura ambiente después de haber calentado las muestras a las temperaturas indicadas

Table II. Room temperatura Vickers microhardnes as a funtion of the annealing temperatura

\begin{tabular}{lll}
\hline & $\mathbf{T}(\mathbf{K})$ & \multicolumn{1}{c}{$\mathbf{H v}$} \\
\hline templado & 350 & $128 \pm 6$ \\
& 550 & $148 \pm 7$ \\
deformado & 663 & $225 \pm 10$ \\
& 350 & $215 \pm 9$ \\
& 550 & $208 \pm 8$ \\
& 783 & $125 \pm 6$ \\
\hline
\end{tabular}




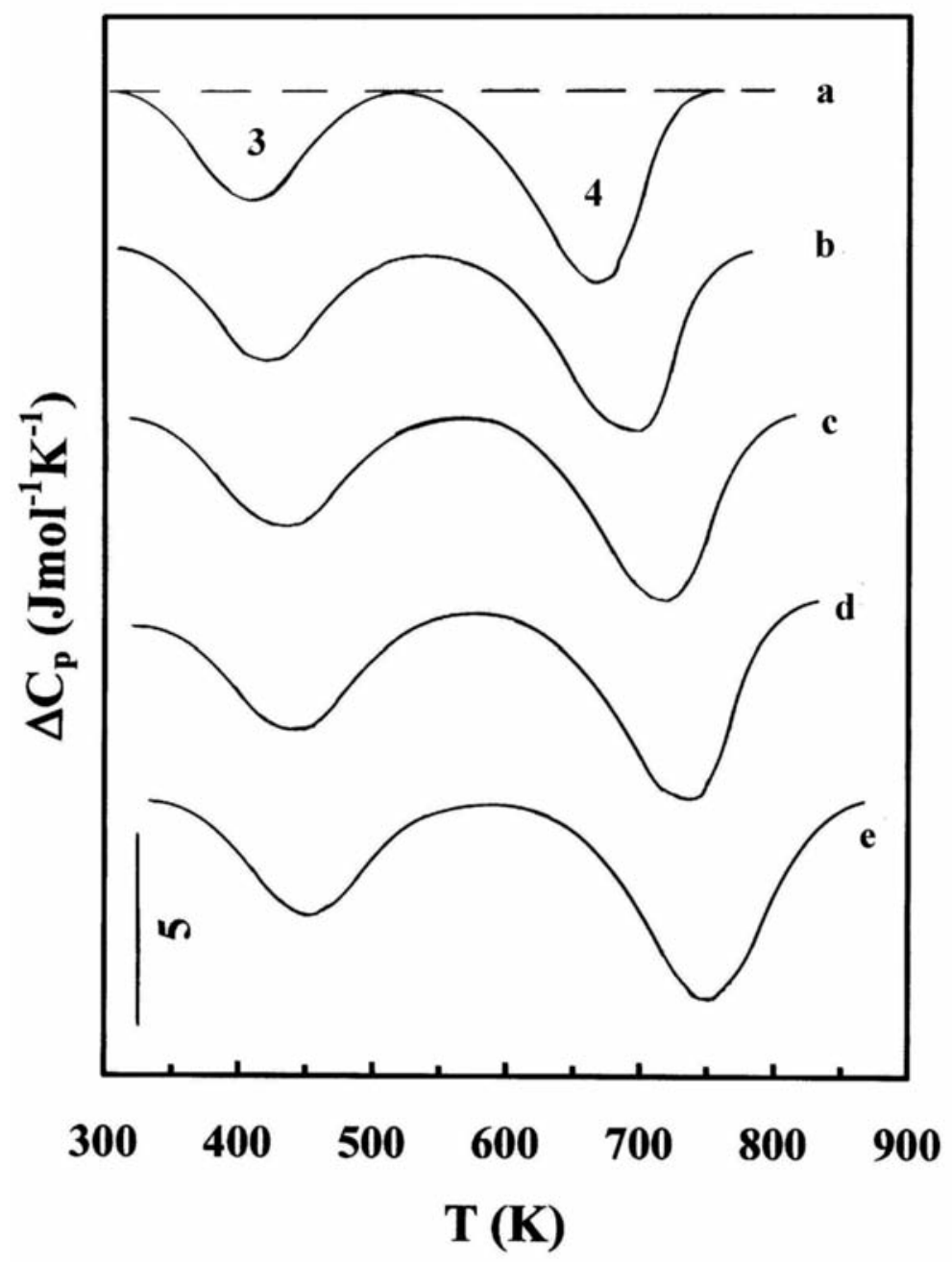

Figura 3. Curvas DSC para Cu- $9 \%$ Ni- $-5,5 \%$ Sn deformado en frío $50 \%$, a las velocidades de calentamiento lineal de: a) $0,083 \mathrm{Ks}^{-1}$, b) $0,167 \mathrm{~K} \mathrm{~s}^{-1}$, c) $0,333 \mathrm{~K} \mathrm{~s}^{-1}$, d) $0,50 \mathrm{~K} \mathrm{~s}^{-1}, \mathrm{y}$ e) $0,667 \mathrm{Ks}^{-1}$.

Figure 3. DSC curves for $50 \%$ cold-rolled Cu-9 \% wt Ni-5.5 wt $\mathrm{Sn}$, at the heating rates: of a) $0.083 \mathrm{Ks}^{-1}$, b) $0.167 \mathrm{~K} \mathrm{~s}^{-1}$, c) $0.333 \mathrm{~K} \mathrm{~s}^{-1}$, d) $0.50 \mathrm{~K} \mathrm{~s}^{-1}$, and $0.667 \mathrm{Ks}^{-1}$.

otra parte, el hecho de que la dureza de la aleación deformada no se modifique apreciablemente al calentarlas en el rango de temperaturas en que se observa el primer pico exotérmico (etapa 3 ) indica que no ha tenido lugar la formación de precipitados durante esta etapa. Este comportamiento podría interpretarse considerando que las dislocaciones parciales generadas por la laminación en frío inhiben la formación de precipitados ${ }^{[15-20]}$ y favorecen la segregación del soluto hacia las dislocaciones parciales. El hecho de que no haya tenido lugar una variación apreciable de la dureza durante el primer pico exotérmico (etapa 3 ) sugiere que la segregación de soluto hacia las dislocaciones solo ha contribuido al anclaje de las mismas sin producir un aumento de la concentración de las dislocaciones. La extraordinaria disminución de la dureza observada durante el segundo pico exotérmico (etapa 4) de la aleación deformada, sugiere que este pico debe estar asociado a la recristalización del material con la consiguiente eliminación de las dislocaciones y de la energía extra asociada a las mismas, al tratarse de defectos en no equilibrio. Un estudio comparativo de la entalpía de la etapa 3 determinada por DSC con la calculada a partir de estimaciones teóricas suponiendo la segregación del soluto hacia las dislocaciones, podría contribuir a esclarecer si dicho proceso es el responsable del primer pico exotérmico (etapa 3 ) registrado para la aleación deformada. 


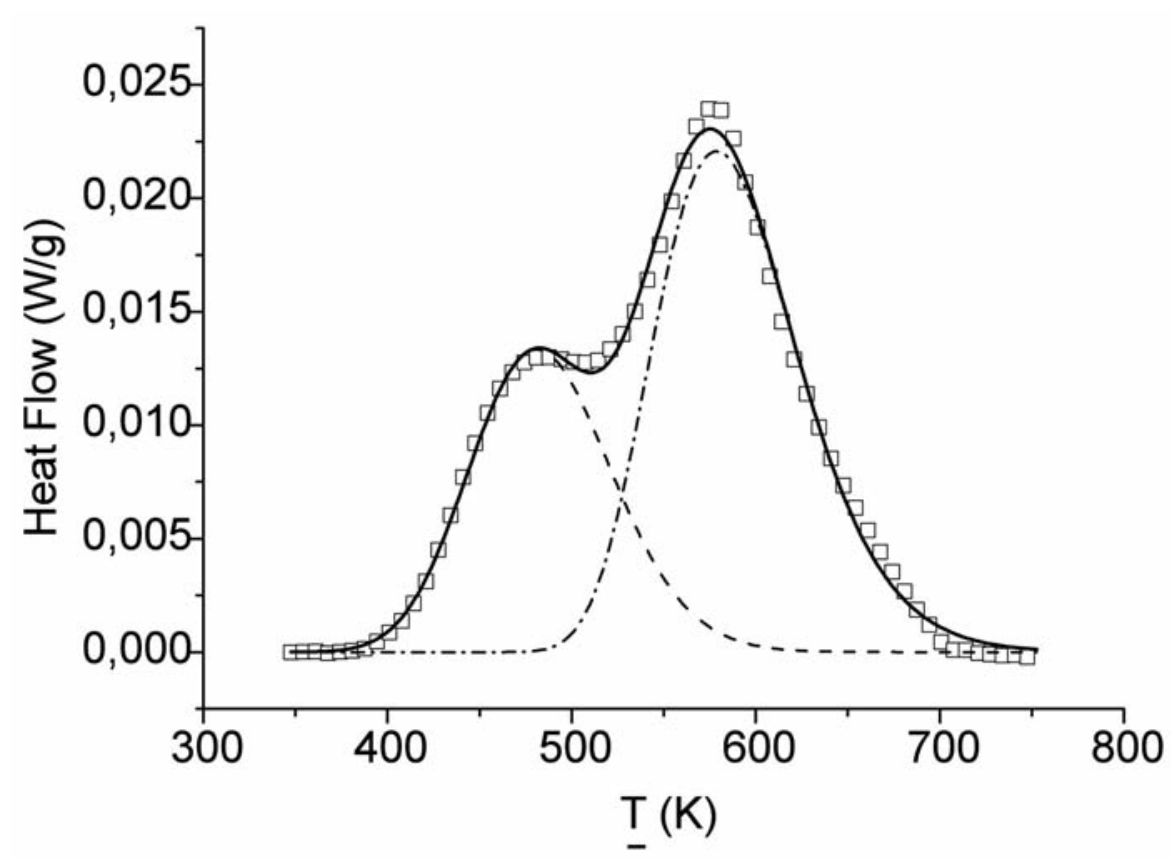

Figura 4. Deconvolución de las etapas 1 y 2 de la figura 1, a la velocidad de calentamiento lineal de $0,167 \mathrm{Ks}^{-1}$, mediante el método de Perejón et.al. ${ }^{[22]}$. (口) datos experimentales, (-) deconvolución, (---) primer proceso, (--) segundo proceso.

Figure 4. Deconvolution of the stages 1 and 2 from the figure 1, at the heating rates of $0.167 \mathrm{Ks}^{-1}$, by Perejón et.al. ${ }^{[22]}$ method. ( $\square$ ) experimental data, (-) deconvolution, (---)firstprocess, (--) secondprocess.

\subsection{Segregación de átomos de soluto hacia las dislocaciones}

De acuerdo a trabajos anteriores ${ }^{[15-20]}$, el calor generado debido a un proceso de segregación de soluto hacia las dislocaciones parciales puede ser expresado por la ecuación (1):

$$
\Delta H_{d}=2 \frac{\rho b^{2}}{c} \int_{0}^{\pi / 2} f(\varphi)\left[\Delta H_{a 1}(\varphi) c_{d 1}(\varphi)+\Delta H_{a 2}(\varphi) c_{d 2}(\varphi)\right] d \varphi
$$

donde $\rho$ es la densidad de dislocaciones, $b$ es el vector de Burger, $c$ es la composición de la aleación, $f(\varphi)$ es la función de densidad de probabilidad de una dislocación de carácter $\varphi, \Delta H_{a 1}(\varphi)$ y $\Delta H_{a 2}(\varphi)$ son las energías de interacción máxima de ajuste entre un átomo de soluto y las componentes parciales de borde de las dislocaciones de borde y helicoidales respectivamente, $c_{d 1}(\varphi)=c \exp \left(-\Delta H_{a 1} / R T\right)$ y $c_{d 2}(\varphi)=c \exp \left(-\Delta H_{a 2} / R T\right)$ son los valores de equilibrio de las concentraciones del soluto en las dislocaciones, $T$ es la temperatura absoluta y $R$ la constante de los gases. Si se considera que c es la concentración de la aleación debido a los solutos níquel y estaño, y se asume que en el material deformado las dislocaciones de borde y helicoidales están presentes en igual cantidad ${ }^{[16,17 \text { y } 20]}, \Delta \mathrm{H}_{d}$ está dado por $^{[16-21]}$ la ecuación (2):

$$
\Delta \mathrm{H}_{\mathrm{d}}=\frac{\pi \rho b^{2}}{\mathrm{c}}\left[\Delta \mathrm{H}_{\mathrm{ae}} \mathrm{c}_{\mathrm{de}}+\Delta \mathrm{H}_{\mathrm{as}} \mathrm{C}_{\mathrm{ds}}\right]
$$

$\Delta H_{a e}$ y $\Delta H_{a s}$ son las energías de interacción de los átomos de níquel y estaño con las dislocaciones parciales, $c_{d e} y c_{d s}$ son las concentraciones de equilibrio a las parciales. Los subíndices e y $s$ se refieren al carácter de la dislocación, de borde y helicoidal respectivamente. Las energías de interacción $\Delta H_{a}$ con una componente de borde $b$, puede calcularse a partir de ${ }^{[26]}$ la ecuación (3):

$$
\Delta H_{a}=\frac{2^{1 / 2}(1+v) b_{e}}{2(1-v) r} G b^{3} e_{a} \sin (\varphi)
$$

donde $b$ es igual a $3^{1 / 2} b / 6$ para la componente parcial de borde correspondiente a una dislocación disociada helicoidal e igual a $0,5 b$ para la componente parcial de borde correspondiente a una dislocación disociada de borde. G es el módulo de cizalla y $e_{a}=d \ln (a) / d c$, es el cambio del logaritmo neperiano del parámetro de red con la concentración de soluto cuando $c \rightarrow 0$ y $v(=0,34)$ es la razón de 
Poisson. La energía de interacción de un átomo de soluto con las componentes parciales helicoidales tanto para una dislocación disociada de borde o helicoidal es $\Delta H_{a}=0$.

La densidad de dislocaciones $\rho$ puede ser evaluada a partir del pico de recristalización como ${ }^{[16-20]}$ la ecuación (4):

$$
\frac{\Delta H_{r} \rho_{s}}{\rho}=\frac{G b^{2}}{4 \pi N} \ln \frac{m}{b \rho^{1 / 2}}+\frac{G b^{2}}{10}
$$

donde $\Delta H_{r}$ es la energía asociada al pico de recristalización, $\rho_{s}\left(=8,86 \times 10^{3} \mathrm{~kg} \mathrm{~m}^{-3}\right)$ es la densidad del material, $N$ y $m$ constantes cuyos valores se estiman en 0,85 y 4 respectivamente ${ }^{[20 \text { y } 21]}$.

Si se considera $b=5,5 \times 10^{-10} \mathrm{~m}$ (determinado a partir del parámetro de red de la aleación $a=2^{1 / 2} b / 2$ ) y $\mathrm{G}=52,1 \mathrm{GPa}$, y que $\Delta \mathrm{H}_{r} \approx \Delta \mathrm{H}_{4}$ (Tabla 1 ), se calcula $\rho=2 \times 10^{15} \mathrm{~m}^{-2}$, a partir de la ecuación (4). El valor promedio de $\rho$ obtenido está en perfecta concordancia con los aportados en la literatura para aleaciones altamente deformadas ${ }^{[27-29]}$.

Con el valor de la densidad de dislocaciones, y mediante las ecuaciones (2) y (3) se evaluaron las energías $\Delta H_{d}$ asociadas a la segregación de átomos de soluto hacia las dislocaciones parciales. El valor promedio de $\Delta H_{d}\left(=428 \pm 9 \mathrm{Jmol}^{-1}\right)$ resultó ser consistente con el valor promedio de las entalpías de reacción de la etapa 3. Estos resultados, conjuntamente con las medidas de microdureza, permiten concluir que la etapa 3 corresponde a un proceso de segregación de átomos de níquel y estaño a las dislocaciones parciales.

\subsection{Análisis cinético}

Las energías de activación $E$ de las diferentes reacciones, necesarias para realizar el análisis cinético, fueron evaluadas a partir de la ecuación de Kissinger modificada ${ }^{[30]}$, (Ec. (5)):

$$
\ln \left(\frac{T_{p}^{2}}{\beta}\right)=\frac{E}{R T_{p}}+\ln \left(\frac{E}{R A}\right)
$$

donde $T_{p}$ es la temperatura del pico (temperatura a la cual la velocidad de reacción es máxima), $R$ la contante de los gases y $\beta$ la velocidad de calentamiento lineal. A partir de las curvas $\ln \left(T^{2}{ }_{p} / \beta\right)$ versus $1 / T_{p}$, mostradas en la figura 6 , se pueden evaluar la energía de activación $E$ y el factor pre-exponencial de

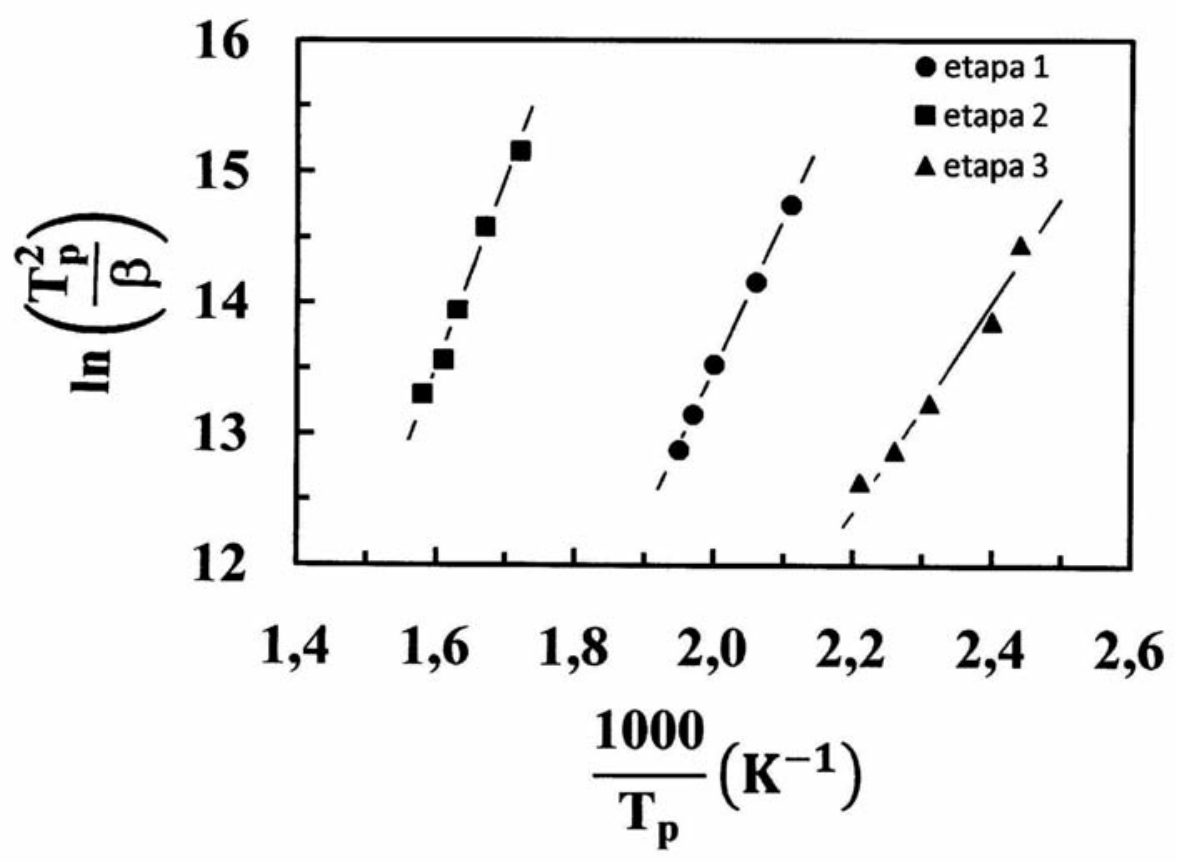

Figura 5. Gráfico de Kissinger modificado para evaluar la energía de activación y el factor pre-exponencial para las aleaciones templada (etapas 1 y 2 ) deformada (etapa 3).

Figure 5. Modified Kissinger plot for evaluating activating energy and pre-exponential factor for the quenched (stages 1 and 2) and cold-rolled (stages 3) alloys. 
Arrhenius A. Los valores correspondientes a $E$ y la constante A se muestran en la tabla I. Los valores de las energías de activación obtenidos para las etapas 1 y 2 resultaron más bajos que las energías de difusión de $\mathrm{Ni}$ en $\mathrm{Cu}\left(=204,3 \mathrm{kJmol}^{-1}\right)$ y $\mathrm{Sn}$ en $\mathrm{Cu}$ $\left(=195,5 \mathrm{kJmol}^{-1}\right)$ estimadas a partir de las correlaciones de Brown y Ashby ${ }^{[31]}$, lo cual puede ser atribuido a la fuerte contribución de las vacantes introducidas por el temple. Por otra parte, el valor de la energía de activación para la etapa 3 resultó mucho más bajo que el requerido para la interdifusión de níquel y estaño en cobre.

El análisis cinético se realizó empleando la ecuación usual de Avrami-Erofeev, utilizada para reacciones heterogéneas, bajo condiciones no isotermas, (Ec. (6)):

$$
\alpha=1-\exp \left[-(A \theta)^{n}\right]
$$

donde $\alpha$ es la fracción reaccionada (obtenida experimentalmente a partir de las curvas calorimétricas) y $\theta\left(=T^{2} R / \beta E \exp [-E / R T]\right)$ es el tiempo reducido ${ }^{[32]}$. A partir de gráficos $\ln \ln (1 / 1-\alpha)$ frente $\ln (\theta)$, se evaluó $n$ de la pendiente de las curvas, cuyos valores se muestran en la tabla I. De acuerdo con la literatura ${ }^{[33]}$, los valores de $n$ cercanos a 1 y 1,5 (etapas 1 y 2 ), resultan compatibles con un proceso en el que interviene nucleación y crecimiento de precipitados, mientras que el valor de $n(=0,6)$ que resulta para la etapa 3, se asocia con la que es de esperar para un proceso de segregación de átomos de soluto hacia las dislocaciones.

\section{CONCLUSIONES}

- El análisis cinético y calorimétrico de los diagramas de DSC obtenidos a diferentes velocidades de calentamiento junto a las determinaciones de microdureza realizadas permiten concluir que el recocido de muestras templadas de una aleación $\mathrm{Cu}-9 \% \mathrm{Ni}-5,5 \% \mathrm{Sn}$ induce su endurecimiento por precipitación de fases de composición $\left(\mathrm{Cu}_{\mathrm{x}} \mathrm{Ni}_{1-\mathrm{x}}\right)_{3} \mathrm{Sn}$. Asimismo, se puede concluir a partir de los resultados de DSC y las medidas de microdureza que las dislocaciones generadas por laminación en frío inhiben el endurecimiento por precipitación favoreciendo la segregación del níquel y el estaño disuelto en la matriz de cobre hacia las dislocaciones. La recristalización de la aleación laminada en frío por recocido de las dislocaciones tiene lugar en una etapa posterior a la segregación del soluto

\section{Agradecimientos}

Los autores desean agradecer al Fondo Nacional de Desarrollo Científico y Tecnológico (FONDECYT), Proyecto № 1110007 , por el apoyo financiero, al Instituto de Ciencias de Materiales de Sevilla, y al Departamento de Ciencia de los Materiales, Facultad de Ciencias Físicas y Matemáticas de la Universidad de Chile por las facilidades otorgadas para desarrollar este estudio.

\section{REFERENCIAS}

[1] J. Miettinen, Computer Couplin of Phase Diagrams and Thermochemistry 27 (2003) 309-318.

[2] B. Alili, D. Braday y P. Zieba, Mat. Charact. 59 (2008) 1.526-1.530.

[3] P. Sahu, S.K. Pradham y M. De, J. Alloys Comp. 377 (2004)103-116.

[4] E.G. Baburaj, U.D. Kulkarni, E.S.K. Menon y R. Krishnan, J. Appl. Cryst. 12 (1979) 476-480.

[5] P. Kratochvil, J. Mencl, J. Pesickay y S.N. Kominik, Acta Metall. 32 (1984) 1.493-1.497.

[6] P. Kratochvily J. Pesicka, J. Mater. Sci. 24 (1989) 2.433-2.436.

[7] P. Virtaneny y T. Tiainen, Mater.Sci. Eng. A 238 (1997) 407-410.

[8] P. Virtanen, T. Tiaineny y T. Lepistö, Mater. Sci. Eng. A251 (1998) 269-275.

[9] J.C. Zhaoy y M.R. Notis, Scripta. Metall. 39 (1998) 1.509-1.516.

[10] J.C. Rhu, S.S. Kim, Y.C. Jung, S.Z. Hany y C.J. Kim, Metall. Trans. A 30 (1999) 2.649-2.657.

[11] N. Lourenço y H. Santos, J. Mater. Eng. Perform. 14 (2005) 480-486.

[12] F. Sadiy C. Servant y J.Thermal, Anal. Cal. 90 (2007) 319-323.

[13] E. Donoso, M.J. Diánez, J.M. Criado, A. Perejón, M.J. Sayagués y L.A. Pérez-Maqueda, $11^{\text {th }}$ Interamerican Congress on Microscopy - CIASEM, Mérida-Mexico, 2011, M-MA13.

[14] F.R.N. Nabarro, Acta Metall. Mater. 38 (1990) 161-164.

[15] M. Militzer, W.P. Sun y J.J. Jonas, Acta Metall. Mater. 42 (1994) 133-141.

[16] A. Varschavsky y E. Donoso, Mater.Sci. Eng. A 251 (1998) 208-215.

[17] A. Varschavsky y E. Donoso, J. Thermal Anal.Cal. 76 (2004) 853-870.

[18] A. Varschavsky y E. Donoso, J. Thermal Anal. Cal. 57 (1999) 607-622.

[19] E. Donoso y A. Varschavsky, Rev. Metal. 35 (1999) 33-38. 
[20] A. Varschavsky y E. Donoso, J. Thermal Anal. 50 (1997) 533-545.

[21] E. Donoso y A. Varschavsky, Rev. Metal. 33 (1997) 3-9.

[22] A. Varschavsky y E. Donoso, J. Thermal Anal.Cal. 8 (2002) 231-241.

[23] A.Varschavsky y E. Donoso, Materials Lett. 57 (2003) 1.266-1.271.

[24] A. Varschavsky y E. Donoso, Mater.Sci. Eng. A145 (1991) 95-107.

[25] A. Perejón, P.E. Sánchez-Jiménez, J.M. Criado y L.A. Pérez-Maqueda, J. Phys. Chem. 115 (2011) 1.780-1.791.

[26] J.D. Eshelby, "Physics of metals-defects", P.B. Hirsch (Ed.), Univ. of Cambridge Press, 1975.
[27] B. Viguier, Mater. Sci. Eng. A349 (2003) 132-135.

[28] A. Rohatgi y K.S. Wecchio, Mater. Sci. Eng. A328 (2002) 256-266.

[29] H.P. Stüwe, A.F. Padilha y F. Siciliano Jr., Mater. Sci. Eng. A333 (2002) 361-367.

[30] E.J. Mittemeijer, L. Cheng, P.J. Van der Shaaf, C.M. Brakmany y B.M. Korevaar, Metall.Trans. A 19 (1988) 925-932.

[31] A.M. Brown y M.F. Ashby, Acta Metall. 28 (1980) 1.266-1.271.

[32] T. Ozawa, J. Thermal Anal. 9 (1976) 369-373.

[33] J.W. Christian, "The theory of transformations in metals and alloys. Part I: Equilibrium and general kinetics theory", Pergamon Press, 1975, pp. 542. 